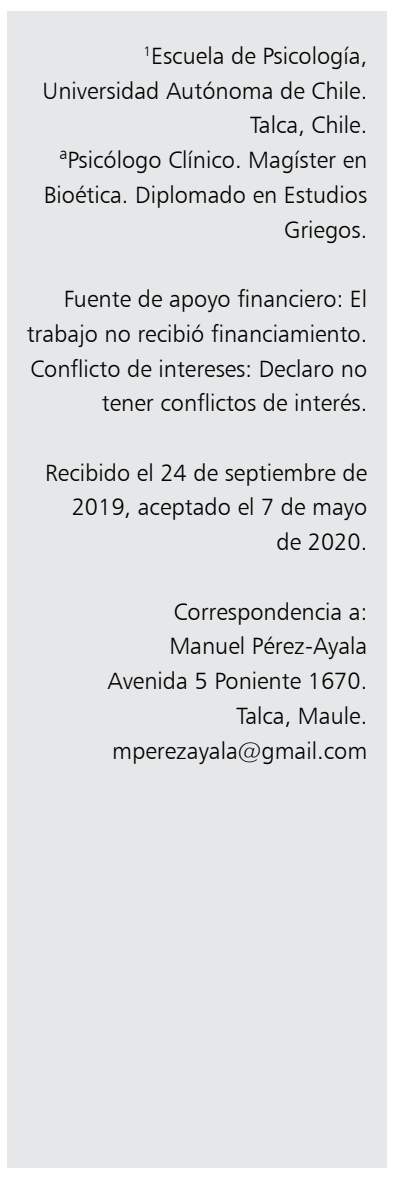

\section{Consideraciones culturales y etimológicas sobre el origen del concepto fobos y su utilización en el contexto clínico y social}

\author{
MANUEL PÉREZ AYALA ${ }^{1, a}$
}

\section{Cultural and etymological considerations on the origin of the phobos concept and its use in the clinical and social context}

The concept of phobia, coming from the Hellenic language, is today widely spread. However, its clinical and social use has significant differences that distort it and induce an erroneous interpretation. Originally associated with fear, it has now become related to hatred. To illustrate this misunderstanding, the case of homophobia is presented. This paper reviews the etymology of the term and its subsequent inclusion in the clinical and social area. With Greek language as parameter, this article proposes terms that describe in a correct and accurate way what is meant to be expressed. The correct use of language is the best manner to investigate, understand and deal with this phenomenon.

(Rev Med Chile 2020; 148: 1004-1010)

Key wods: Gender Identity; Language; Psychology; Sexual Behavior; Terminology as Topic.

\footnotetext{
E
} s natural que las palabras varíen y muten desde sus concepciones originales. En el caso del español, derivado principalmente del griego y latín, términos de uso habitual en ciencias $\mathrm{u}$ otras disciplinas formales suelen adquirir usos populares en la comunicación. Las diversas culturas hacen suyos los conceptos y cada generación les dota de especial significación. En el caso del término fobos, su utilización clínica guarda una fidedigna relación con su noción primaria, pero se evidencia que su empleo coloquial es inexacto, donde más que un estricto apego, se ha extendido sólo una representación parcial. Tal distorsión finalmente induce al error interpretativo del fenómeno que se desea expresar y representar.

Este artículo no pretende un abordaje mutidimensional exhaustivo. Su propósito principal es plantear observaciones fundamentadas sobre la corrección conceptual, valiéndose para esto del análisis etimológico y la revisión del sentido y aplicación asignada, primero, en el contexto clínico de salud, y luego cómo ha sido propuesto e inexactamente empleado en el ámbito socio-cultural. Las sugerencias se dirigen hacia quiénes se desempeñan en el amplio rango del ámbito profesional como una invitación a reexaminar los discursos colectivamente elaborados y contribuir a precisar el dominio de la palabra y la práctica del lenguaje.

\section{Historia y etimología del concepto fobos}

Comenzando en las inscripciones cretense-micénicas de los siglos XIII y XII a. C. hasta el presente, la lengua griega no ha perdido continuidad. A los treinta y cuatro siglos de tradición oral y escrita, se debe añadir los siglos de tradición so- 
lamente oral. Con la única excepción de la lengua china, ninguna otra lengua actual posee tan larga e ininterrumpida tradición ${ }^{1}$. Desde su convergencia lingüística a raíz de los distintos dialectos que le dieron forma a través del proceso que implica desarrollo, codificación, aceptación y elaboración de una norma ${ }^{2}$, los cambios que la lengua helénica experimentó en todos sus siglos de vida no lograron alterar su esencia ni convertirla en otra lengua. Su forma presente sintetiza un producto natural de incesante evolución, que ahora permite reflexionar sobre diferentes facetas de su sociedad, así como de su vida cotidiana ${ }^{3}$.

Originario de su historia y cultura, Fobos, como sustantivo propio, es nombrado cuatro veces en la obra épica cumbre de la cultura occidental, Ilíada, (Canto XI, verso 37; Canto XIII, verso 299; Canto $\mathrm{XV}$, verso 119), y su primera aparición (Canto IV, verso 440), es a propósito de la incitación a la disputa entre aqueos y troyanos. Homero, en la traducción de Luis Segalá y Estalella ${ }^{4}$, refería: “ $A$ unos, los exitaba Ares; a los otros, Atenea, la de los brillantes ojos, y a entrambos pueblos, el Terror, la Fuga y la Discordia" (Fobos, Deimos y Enio). Otras traducciones de la misma obra les nombran igual ${ }^{5}$, o como Terror, Huida y Disputa ${ }^{6}$.

Luego, en su Teogonía, Hesíodo, en la traducción de Aurelio Pérez Jiménez ${ }^{7}$, al describir las descendencias olímpicas, señala (versos 935-938): "A su vez, con Ares, perforador de escudos, Afrodita concibió a los temibles Miedo y Terror, que ponen en confusión las compactas falanges de varones en la guerra sangrienta junto con Ares destructor de ciudades". Para Homero -en interpretación de sus traductores- las concepciones de miedo y terror eran prácticamente lo mismo, algo que sí distingue diferencia en Hesíodo.

Según la mitología griega, Fobos, hijo de Ares y Afrodita -dioses de la beligerancia y la belleza respectivamente-era la personificación del miedo. Aparecía antes de cada batalla infundiendo espanto a los combatientes que se aprestaban a luchar, quienes huían despavoridos o fingían su muerte para luego escapar. Como horror, se asociaba a la parálisis ante la contemplación de algo que es inevitable evadir. Su equivalente en la tradición romana fue Timor, de donde deriva nuestro vocablo temor. Se manifestaba acompañado de su hermano Deimos -el dolor o angustia que podía alterar e incluso trastornar a las personas-y su hermanastra Enio, asociada a la destrucción y masacres.
Investigada su etimología, disciplina que trata sobre cómo se produjeron las palabras desde las antiguas raíces y su original significación, composición y evolución ${ }^{8}$, uno de los más reputados expertos en la lengua griega, el lingüista francés Pierre Chantraine ${ }^{9}$, refiere que la palabra $\phi o ß o s$ (fobos) existe ya en Homero, y no hay cómo rastraer su origen antes del famoso rapsoda. Señala que el verbo $\phi \varepsilon \beta о \mu \alpha \imath$ (febomai) significaba huir por pánico, o por terror, y que esta conjugación sólo está atestiguada en presente imperfecto en los escritos atribuidos al Ciclo Épico ${ }^{10}$; fragmentos de poemas de la Grecia arcaica que trataban sobre tres principales temáticas: a) Gigantomaquia, que relata el origen del mundo (cosmogonía) y la lucha de Zeus contra los gigantes; b) Ciclo Tebano, que narra la leyenda de Edipo y las luchas de sus descendientes por la ciudad; y c) Ciclo Troyano, que describe desde el origen del problema que motiva la guerra por la ciudad, la lucha y muerte de los principales héroes, hasta el retorno de los sobrevivientes.

El significado de fobos como huida por miedo sólo aparece así en Homero y en los poetas épicos; después se traduce únicamente como miedo. Es decir, fobos, en la épica griega tiene la doble acepción de fuga por pánico. Luego, desde el séptimo siglo a. C en adelante, sólo se le entenderá como miedo, no necesariamente vinculado a huida.

En síntesis, fobos y su familia léxica representa el miedo desmesurado, el horror ante lo que de manera indefectible se debe enfrentar, y que sin embargo únicamente se desea huir. El concepto clínico fobia al que debe su origen acierta en el sentido de su uso. Fuera de ésta área se le atribuyeron otras características y connotaciones que van más allá de su representación original, y que conviene aclarar para su mejor comprensión y, en otros casos, su expresión a través de términos distintos.

\section{Fobia como concepto clínico: distinciones entre miedo, angustia y ansiedad}

Como manifestación psciopatológica, la revisión de la fobia abarca planos semiológicos, nosológicos y explicativos. Antes de su utilización en el área clínica, se debatió sobre la necesidad de diferenciar al miedo de conceptos afines. La discusión para distinguir el solapamiento incorporó 
primero a la angustia en el plano filosófico y luego a la ansiedad, en el psicopatológico.

Desde la reflexión filosófica, para el danés Søren Kierkegaard ${ }^{11}$, el primer autor moderno en convertirla en materia central de su obra, la angustia es propia de la experiencia existencial, y emerge como una amenaza manifestada en el temor a morir, a perder la cordura, o ante la nada, simbolización del vacío existencial. El origen de la angustia se encuentra en la posibilidad de equivocarse o de no lograr el objetivo propuesto por el proyecto vital. Lo que Kierkegaard llama posibilidad -equivalente de libertad-, vincularía a la angustia con lo indeterminado. El objeto de la angustia, la nada, involucra una dimensión temporal en ella. La angustia, sostiene, es un experiencia humana, singular y excepcional, producto exclusivo de la condición como tal.

Continuando esa línea, el psiquiatra y filósofo alemán Karl Jaspers ${ }^{12}$, explorador de la relación entre filosofía y psicopatología, en su búsqueda de establecer principios que organizaran y diferenciaran las manifestaciones de la vida psíquica, propone una distinción en la categoría sentimientos sin objeto: mientras que la angustia es atópica, el miedo es dirigido a algo (p. 130). Desde la separación entre angustia y miedo realizada por Jaspers (miedo referido a la presencia de un objeto en tanto percepción o representación, y angustia, ligada a la ausencia de éste), ambos se instalan en la escena médica para referirse a un estado mental: si bien es una experiencia humana comprensible, también podía dar lugar a un cuadro patológico ${ }^{13}$, idea prolongada por el neurólogo austríaco y fundador del psicoanálisis Sigmund Freud. Para éste $^{14}$, angustia refiere a un estado y prescinde del objeto, mientras que miedo dirige la atención justamente al objeto.

El mismo Freud se ocupó del concepto fobia, siéndole complejo separarla de la angustia, entendiendo que la verdadera fobia se asocia incondicionalmente a simbolización y proyección, descargando al mundo interno de lo que es ajeno e insoportable. En una temporalidad específica, expresa la primera respuesta hacia la representación del peligro o amenaza vital. La fobia no hablaría tanto del miedo sino de la angustia, pero de esa angustia de la cual el sujeto busca las circunstancias en que surge para librarse de ella. La condición que posibilitaría la fobia sería la fragilización del sentimiento de existencia, con un impedimento del pensar debido a la amenaza de desmoronamiento psíquico ${ }^{15}$.

Dispuestos como objetos de interés y estudio $^{16}$, la literatura distingue entre miedo y angustia, utilizando miedo para la actitud hacia un peligro que amenaza desde lo real y angustia para las reacciones ante amenazas que surgen del mundo interno. La expresión psicopatológica explica al miedo como fenómeno evolutivo normal, y a las fobias como síntomas ${ }^{17}$. Entendida como un patrón primitivo, fobia es primariamente definida como un miedo persistente a un objeto, actividad o situación, donde la persona es capaz de reconocer que el miedo es excesivo e irracional en proporción a la peligrosidad real de este objeto, actividad o situación, produciendo además ansiedad desbordante, conductas de evitación e interferencia significativa en la vida cotidiana $^{18,19}$.

Las investigaciones posteriores en psicopatología fueron vinculando de manera progresiva a la fobia con la ansiedad, al punto de hoy circunscribirla en su clasificación. Si bien la ansiedad sería cercana al miedo, se diferencia de éste en que, mientras el miedo es una perturbación cuya presencia se manifiesta ante estímulos presentes, la ansiedad se relaciona con la anticipación de peligros futuros e indefinibles ${ }^{20}$. El DSM $5^{21}$ define a la fobia específica como el "miedo o ansiedad intensa por un objeto o situación específica” (pp. 197-199), y propone que se clasifique según el tipo de estímulo fóbico que le origina, que puede ser animal, del entorno natural, sangre-inyección-herida, situacional u otra. Los criterios diagnósticos principales son básicamente dos: la provocación de ansiedad y la evitación o la resistencia con miedo intenso presentes un mínimo de seis meses.

Clínicamente, las fobias se experimentan con malestar y displacer que incluye altos niveles de ansiedad y ocasiona deterioro en varias áreas de la vida de quién le experimenta, con el elemento clave de respuesta fóbica o evitativa. Las discusiones que elaboraron las diferencias principales entre miedo, angustia y ansiedad para comprender y delimitar las fobias en el campo clínico, tienen un correlato directo con el concepto fobos original. Esto no sucede así en el ámbito socio-cultural. Para ejemplificar este punto, se revisará la construcción y empleo del concepto homofobia. 


\section{Utilización del concepto fobia en el contexto socio-cultural: el caso de la homofobia}

La homofobia fue el primero de una serie de fenómenos sociales relacionados a la denominada diversidad sexual estudiados durante las últimas cuatro décadas, junto a la lesbofobia y transfobia $^{22-24}$. La introducción del término homofobia por parte del Psicólogo George Weinberg durante la década de 1960 desafió el pensamiento tradicional sobre la homosexualidad y ayudó a centrar la atención de la sociedad cuestionando la normalización del estigma antigay. Luego, el sociólogo francés Daniel Welzer-Lang ${ }^{25}$ amplió el espectro conceptual incorporando a los discursos y comportamientos en forma de hostilidad respecto a prácticas opuestas a los roles preestablecidos, como una manifestación del sexismo y la discrimación de personas en razón de su sexo (macho/ hembra), y más concretamente de su género (masculino/femenino).

Estudiada de manera teórica y empírica ${ }^{26}$, aún cuando la homofobia como constructo se validó por estudios conductuales en los cuales se midieron respuestas somáticas equivalentes a las fobias, verificando que el homonegativismo tiene un efecto biológico, por ejemplo, con alteración del ritmo cardíaco ${ }^{27}$, la mayoría de las situaciones que actualmente refieren a la homofobia lo hacen respecto al rechazo asociado al odio y al prejuicio ${ }^{28}$.

Sin desestimar la importancia de la visibilización e impacto que ha significado el término, expertos discuten las limitaciones y la distorsión a la que induce ${ }^{29}$, incluyendo la suposición de que el prejuicio antigay se basa principalmente en el miedo y una incapacidad social que de cuenta de los cambios históricos en la forma de considerar la homosexualidad como identidad. Uno de esos expertos que evidenció la necesidad de un nuevo vocabulario para avanzar en su elaboración es el Psicólogo Gregory Herek ${ }^{30}$. Postula que el término homofobia supone incorrectamente que las actitudes negativas hacia las personas pertenecientes a la diversidad sexual se basan en un miedo patológico e irracional -fobia-, mientras que las investigaciones que ha liderado indican que se le consideran con mayor precisión como una forma de prejuicio.

Para Herek, prejuicio sexual es el término preferible a homofobia porque refiere a las actitudes negativas hacia un individuo debido a su orientación sexual y no transmite ninguna suposición sobre las motivaciones que subyacen a las actitudes negativas. Propone tres términos para la mejor compresión del fenómeno ${ }^{31}$ : a) prejuicio sexual, actitudes negativas de los individuos basadas en la orientación sexual; b) estigma sexual, conocimiento compartido de la consideración negativa de la sociedad hacia cualquier comportamiento, identidad, relación o comunidad no heterosexual); y c) heterosexismo, ideología cultural que perpetúa el estigma sexual.

Homofobia hasta hoy es utilizada para referirse a la actitud negativa u hostil hacia personas homosexuales $^{32}$, expresada en diferentes formas activas de violencia física, verbal y simbólica, análoga a otras formas de exclusión ${ }^{33}$, y en un rechazo silencioso hacia las personas identificadas como homosexuales, que limita su acceso a derechos, espacios, reconocimiento o poder.

Pese a que el componente clave de la homofobia es el rechazo irracional hacia la homosexualidad, no puede ser reducido sólo a eso ${ }^{34}$. En sentido estricto, una fobia es sinónimo de miedo irracional. En el caso de la homofobia, su rango incluye odio y señalar como contrario, inferior o anormal $^{35}$. Entretejida con el sexismo y el racis$\mathrm{mo}^{36}$, es una manifestación arbitraria que excluye al tercero/a (objeto del desprecio) fuera del ámbito de lo humano. La lógica subyacente a la homofobia es similar a las formas de violencia que comparten como rasgo común la deshumanización.

\section{Propuestas para la precisión terminológica y la correcta expresión}

Según esta revisión, conceptos derivados del ámbito clínico como agorafobia, claustrofobia, acrofobia y, dentro de las zoofobias, aracnofobia, son ejemplos fieles de lo que fobos representa. En cambio, los que provienen del ambiente socio-cultural, aparte de los recién examinados homo, lesbo y transfobia, tales como xenofobia ${ }^{37,38}$, hispanofobia, islamofobia ${ }^{39}$, y el anglicismo de uso reciente fatphobia ${ }^{40-42}$, no aluden a él de manera correcta. Es manifiesto que acuñaciones que originalmente referían al miedo y sus semejantes, ahora se relacionan más con actitudes negativas que no sólo designan repudio, sino que incluso expresan odio. Por esto, la utilización de fobos como sufijo no debe ser entendida análoga en el campo clínico a la etiquetación externa de éste: mientras en el 
primero manifiesta alteración psicológica, en el segundo es sinónimo de intolerancia social.

Si se desea expresar miedo, temor, horror, paralización, e incluso pánico; así como huida o escape por terror, fobos es el concepto correcto. Si, por otra parte, se pretende representar rechazo, repudio, desprecio, aversión, hostilidad, prejuicio y discriminación, fobos no es la palabra que le interprete de manera apropiada.

Tómese, por ejemplo, los vocablos misoginia, misandria o misantropía, relacionados con el odio o prejuicios negativos contra la mujer, el hombre y la humanidad, respectivamente. El prefijo de estas locuciones deriva de la palabra griega misó, del verbo contracto $\mu 1 \sigma \varepsilon \omega / \mu 1 \sigma \omega$; odio (43; p. 906).

En la práctica, ambos conceptos - fobos y misópueden converger en el denominador común del rechazo ocasionado, pero con una significativa diferencia: en el caso de la fobia, esta incita una respuesta evitativa; es decir, de manera activa la persona pretende alejarse, rehuir del estímulo en cuestión. Sería, así, pertinente hablar de conductas o actitudes homomisoicas, xenomisoicas o hispanomisoicas, según el caso, toda vez que incluso pudiendo develar un rechazo por la experiencia de temor psíquico, las fobias, en su concepción clínica, no suelen motivar respuestas agresivas o violentas en contra del estímulo que les origina.

En síntesis, el rechazo en tales casos no promueve evitación o elusión, sino que hostilidad. Por lo tanto, lo que se quiere expresar con este "rechazo" no es el miedo, sino el odio. Lo adecuado sería hablar de homomisia, xenomisia, hispanomisia, y no de homofobia, xenofobia, hispanofobia, si se quiere describir actos que encarnen rechazo o prejuicio por odio. Otra alternativa, incluso más específica, deriva del término griego $\alpha \pi 0 \rho \rho \varepsilon \sigma 1 \varsigma-\varepsilon \omega \varsigma$ (apórresis-eos) (43; p. 190), repudio, recusación; $\alpha \pi \circ \rho \rho \imath \psi \imath \varsigma-\varepsilon \omega \varsigma$ (apórripsis); rechazamiento, repulsión.

Sea homomisia u homoapórresis, xenomisia o xenoapórresis, alguien que padezca alguna fobia, con o sin diagnóstico profesional, no degrada u odia al objeto que encarne el estímulo que la provoca. Personas que padecen cualquier tipo de prejuicio, pueden serlo por rechazo, pero este sería producto de repudio u odio, no de miedo. Como fuere, en todos estos casos no es posible abstraerse de las problemáticas de fondo, que dan cuenta de transgresiones, vulneraciones y perjuicios hacia otros seres humanos.

\section{Consideraciones finales}

Aunque impreciso, el uso cultural del término fobia está socialmente legitimado y colectivamente posicionado. Es presumible su continuidad como tal. Las explicaciones de este fenómeno pueder variar desde el léxico acostumbrado y familiaridad, hasta la relativa facilidad (plasticidad) de conjugación.

Este texto no proyecta zanjar el debate acerca de la utilización del concepto fobia, proponer neologismos ideológicamente controversiales, ni confundir expresiones coloquiales cotidianas con vocablos técnicos, pero sí aportar a la discusión desde su marco histórico y procedencia etimológica, para su revisión como constructo. Es probable que profesionales del área clínica no necesiten una aclaración a este respecto. Menos aún esta precisión sería exigible a las representaciones populares. En caso de rectificación, como interpelación apuntaría al conjunto general de profesionales en sus roles educativos, formativos y normativos, por el deber de divulgación rigurosa y guía ejemplificadora que les cabe en la sociedad.

Han sido varios los autores que no sólo develaron el error, sino que también propusieron terminologías adecuadas y certeras sobre lo que se quiere declarar, como el ya referido prejuicio sexual, o también estigma y homonegatividad, entre las más utilizadas. No se pretende negar ni enmascarar el uso coloquial transversal de los términos que suelen llevar como sufijo "fobia", asociado a actitudes oprobiosas, sino que enmendar $y$ reformar sus conceptualizaciones para que las expresiones deseadas sean acertadas. Garantizar la consideración de la alteridad y respeto al prójimo, inicia en la comprensión, entendimiento, disposición y acuerdo logrado a través del lenguaje.

A su vez, se comprende al lenguaje común no sólo como un ejercicio intelectual, sino de comunicación y expresión que adquiere independencia en la medida que resulta funcional a la interacción social entre individuos. Las palabras, que expresan nuestro pensamiento y guían nuestras acciones, reflejan intención y representan poder. Consensuado por sujetos programados o moldeados por su cultura ${ }^{44}$, el entramado del lenguaje construye realidades, desde un análisis personal del mundo que se habita.

Como expresiones de intolerancia y restricción de libertad, las dos concepciones contrapuestas de 
fobos revisadas: la correcta, respecto al miedo, y la desacertada, relacionada al prejuicio, repudio $\mathrm{u}$ odio, son requirientes de tratamientos psicológicos y/o psiquiátricos especializados, por lo limitante, desadaptativo e incapacitante de sus distintos alcances individuales y sociales.

Agradecimientos: El autor agradece al Profesor Miguel Castillo Didier, Director del Centro de Estudios Griegos, Bizantinos y Neohelénicos, por la guía, revisión y comentarios en la investigación etimológica de la lengua helénica.

\section{Referencias}

1. Clair C. Lengua helénica. Bizantion nea hellás 1971; 2: 179-97.

2. Haugen E. Dialect, Language, Nation. American Anthropologist 1966; 68: 922-35.

3. Rodríguez A. Arqueología de la palabra. Oralidad y escritura en el mundo antiguo. Barcelona, España: Bellaterra; 2010. 304 p.

4. Homero. La Ilíada. Traducción de Luis Segalá y Estalella. Barcelona, España: Edicomunicación; 1994.

5. Homero. Ilíada. Introducción, versión rítmica y notas de Rubén Bonifaz Nuño. Ciudad de México, México: Universidad Autónoma de México; 2005.

6. Homero. Ilíada. Traducción y notas de Crespo, E.; revisión García Gual, C. Madrid, España: Editorial Gredos; 2006.

7. Hesiodo. Teogonía. Traducción y notas de Pérez, A., y Martínez, A. Editorial Gredos: España; 2006.

8. Razís J. Algunas consideraciones etimológicas. Bizantion nea hellás 1991; 11-12: 201-3.

9. Chantraine P. Dictionnaire Étymologique de la Langue Grecque. Histoire des Mots. Paris, France: Éditions Klincksieck; 1968.

10. Pajares A. Fragmentos de épica griega arcaica. Madrid, España: Editorial Gredos; 1979.

11. Kierkegaard S. El concepto de la angustia. Madrid, España: Alianza Editorial, 2013. 320 p.

12. Jaspers K. Psicopatología General. Segunda edición. Mexico: Fondo de Cultura Económica; 1993.

13. Pizarro F. La inscripción de la angustia en el campo psicopatológico: referencias etimológicas, consideraciones filosóficas y categorías precursoras. Rev. Asoc. Esp. Neuropsiq 2011; 31(110): 229-41 doi: 10.4321/S021157352011000200004.

14. Freud S. Conferencias de introducción al psicoanálisis (1916-1917). 25a Conferencia. La angustia. Obras Com- pletas, Vol. XVI. p. 357- 374. Buenos Aires, Argentina: Amorrortu; 1998.

15. Birraux A. La fobia, estructura originaria del pensamiento. Revista Uruguaya de Psicoanálisis 2004; 99: 101-31.

16. Dixon J, de Monchaux C, Sandler J. Patterns of anxiety: the phobias. British Journal of Medical Psychology 1957; 30(1): 34-40 Disponible en: https://doi.org/10.1111/j.2044-8341.1957.tb01941.x

17. Dío E. Temores y Fobias. Condiciones de génesis en la infancia. Barcelona, España: Gedisa Editorial; 1998

18. Marangoni A. El origen de las palabras. Etimología de algunos términos usados en medicina. Revista Argentina de Radiología 2009; 73 (2): 149-52

19. Ollendick T, Raishecich N, Davis T, Sirbu C, Öst L. Specific phobia in youth: Phenomenology and Psychological Characteristics. Behav Ther 2010; 41 (1): 133-41.

20. Sierra J, Ortega V, Zubeidat I. Ansiedad, angustia y estrés: tres conceptos a diferenciar. Revista Mal-estar e Subjetividade 2003; 3 (1): 10-59.

21. American Psychiatric Association. Manual diagnóstico y estadístico de los trastornos mentales (DSM-5) (Quinta edición). Madrid, España: Editorial Médica Panamericana; 2014.

22. Nagoshi J, Adams K. Gender Differences in Correlates of Homophobia and Transphobia. Sex Roles 2008; 59: 521-31. doi: 10.1007/s11199-008-9458-7.

23. Warriner K, Nagoshi C, Nagoshi J. Correlates of Homophobia, Transphobia and Internalized Homophobia in Gay or Lesbian and Heterosexual Samples. Journal of Homosexuality 2013; 60: 1317-34. doi: 10.1080/00918369.2013.806177.

24. Rasmussen M, Sanjakdar F, Allen L, Quinlivan K, Bromdal A. Homophobia, transphobia, young people and the question of responsibility. Discourse: Studies in the Cultural Politics of Education 2015; 1-13. Disponible en: http://dx.doi.org/10.1080/01596306.2015.1104850.

25. Welzer-Lang D. L'homophobie: la face cachée du masculin. In Dans D. Welzer-Lang P. Dutey, and M Dorais (dir.). La peur de l'autre en soi, du sexisme à l'homophobie. Montreal: VLB; 1994. p. 13-92.

26. Gómez F, Barrientos J. Efectos del prejuicio sexual en la salud mental de gays y lesbianas en la ciudad de Antofagasta, Chile. Sexualidad, Salud y Sociedad. Revista Latinoamericana 2012; 10(abr): 100-23.

27. Shields S, Harriman R. Fear of male homosexuality: cardiac responses of low and high homonegativism males. J Homosex 1984; 10 (1-2): 53-67.

28. Barrientos J, Cárdenas M. Homofobia y calidad de vida de Gay y Lesbianas: Una mirada Psicosocial. PSYKHE 2013; 22 (1): 3-14. doi: 10.7764/psykhe.22.1.553.

29. Garrido R, Morales Z. Una aproximación a la Homo- 
fobia desde la Psicología. Propuestas de Intervención. Psicología, Conocimiento y Sociedad 2014; 4 (1): 90115. Disponible en: http://revista.psico.edu.uy/index. php/revpsicologia.

30. Herek G. The psychology of sexual prejudice. American Psychological Society 2000; 9 (1): 19-22 doi:10.1111/1467-8721.00051

31. Herek G. Beyond "homophobia”: Thinking about sexual prejudice and stigma in the twenty-first century. Sexuality Research \& Social Policy 2004; 1 (2): 6-24.

32. Borrillo D. Homofobia. Barcelona, España: Bellaterra; 2001.

33. Cornejo J. Bullying homofóbico en Chile: trayectoria histórica. Límite. Revista Interdisciplinaria de Filosofía y Psicología 2014; 9 (30): 61-70.

34. Cornejo J. Componentes ideológicos de la homofobia. Límite. Revista Interdisciplinaria de Filosofía y Psicología 2012; 7 (26): 85-106.

35. De la Espriella R. Homofobia y Psiquiatría. Revista Colombiana de Psiquiatría 2007; 36 (4): 718-35.

36. Kimmel M. Masculinity as Homophobia: Fear, Shame and Silence in the Construction of Gender Identity. In Feminism and Masculinities, Murphy, P. Ed. Oxford readings in feminism. Oxford, Great Britain: Oxford University Press; 2004.
37. Hjerm M. National Identities, National Pride and Xenophobia: A Comparison of Four Western Countries. Acta Sociologica 1998; 41: 335-47.

38. Cashdan E. Ethnocentrism and Xenophobia: A Cross-Cultural Study. Current Anthropology 2001; 42 (3): 760-65.

39. Mignolo W. Islamophobia/Hispanophobia: The (Re) Configuration of the Racial Imperial/Colonial Matrix. Human Architecture: Journal of the Sociology of Self-Knowledge 2006; 5: 13-28.

40. Sarikaya R, Ozturk H, Afyon Y, Turegun E. Examining university students' attitudes towards fat phobia. Turkish Journal of Sport and Exercise 2013; 15 (2): 70-4.

41. Flint S, Reale S. Obesity stigmatization by obesity researchers. Lancet 2014; 384: 1925-6. doi: 10.1016/ S0140-6736(14)62276-5.

42. Flint S, Hudson J, Lavallee D. UK adults' implicit and explicit attitudes towards obesity: a cross-sectional study. BMC Obesity 2015; 2: 31. doi: 10.1186/s40608015-0064-2.

43. Sebastian F. Diccionario Griego-Español. Barcelona, España: Editorial Ramón Sopena; 1964.

44. Sevilla M, Álvarez N. Normalización del discurso homofóbico: aspectos bioéticos. Acta Bioethica 2006; 12 (2): 211-17. 